\title{
Growth and characterization of pure and doped btzs single crystals
}

\author{
Sethu Gunasekaran ${ }^{1}$, Padmapriya Venkatesan ${ }^{1, *}$, Govindarajulu Anand ${ }^{2}$, \\ Subramanian Kumaresan ${ }^{3}$ \\ ${ }^{1}$ PG and Research Department of Physics, Pachaiyappa's College, Chennai-600 030. India) \\ ${ }^{21}$ Department of Physics, Arulmigu Meenakshi Amman College of Engineering, Vadamavandal - 604410 , \\ India) \\ ${ }^{3}$ Department of Physics, Government Arts College, Stone House Hill, Ooty - 643 002. India)
}

\begin{abstract}
The growth and characterization of pure and doped BTZS single crystals are discussed. In the present study powder X-Ray diffraction data of the grown crystal were recorded using BRUKER D8 Advance powder crystal X-ray diffractometer with $2.2 \mathrm{KW} C \mathrm{Cu}$ anode ceramic X-ray tube. The various functional groups present in the grown crystals were identified and confirmed by recording the FTIR spectrum using BRUKER IFS-66V spectrophotometer by KBr Pellet Technique in the region $4000-400 \mathrm{~cm}^{-1}$. The UV-Vis - NIR optical spectra of the crystals were recorded using the VARIAN CARY 5E model spectrophotometer. The degree of dopant inclusion was estimated by using Atomic Absorption Spectroscopy. The SHG efficiencies of the crystals were studied using Nd:YAG $Q$ - switched laser.
\end{abstract}

Keywords: Atomic Absorption Spectroscopy, FTIR Spectrum, SHG, UV-VIS-NIR optical spectra, X-ray diffraction.

\section{Introduction}

The search for novel non linear optical (NLO) crystals is gaining increased attention in recent years and a wide variety of both organic and inorganic NLO materials has been developed. Problems with both classes of materials have resulted in the investigation of semi organics [1-6]. The semi organic materials have the potentials for combining the high optical non linearity and chemical flexibility of organics with thermal stabilities and excellent transmittance of inorganic [7, 8]. In search of this semi organic NLO materials urea and urea analogs have been explored [9]. Single crystals of Zinc thiourea chloride (ZTC), zinc thioureasulphate (ZTS) and Bisthiourea cadmium chloride(BTCC) were grown and characterized by N.R.Dhumane[10]. J.Ramajothi et.al [11] reported the growth and characterization of tristhiourea zinc sulphate In the present work, attempts have been made to improve the physiochemical properties of BTZS with metal substitution $\left(\mathrm{Cd}^{2+}\right.$ and $\left.\mathrm{Cu}^{2+}\right)$. Pure and metal doped BTZS were grown and characterized by XRD, optical, thermal, mechanical, dielectric and conductivity studies.

\section{Synthesis}

BTZS salt was synthesized by dissolving zinc sulphate (AR grade) and thiourea (AR grade) in the molar ratio 1:2 in double distilled water. Zinc sulphate reacts with thiourea according to the reaction:

$\mathrm{ZnSO}_{4}+2\left[\mathrm{CS}\left(\mathrm{NH}_{2}\right)_{2}\right] \longrightarrow \mathrm{Zn}\left[\mathrm{CS}\left(\mathrm{NH}_{2}\right)_{2}\right]_{2} \cdot \mathrm{SO}_{4}$

The synthesis of $\mathrm{Cd}^{2+}$ and $\mathrm{Cu}^{2+}$ doped BTZS salts were done by replacing $\mathrm{Zn}^{2+}$ in BTZS by 3 mole $\%$ of $\mathrm{Cd}^{2+}$ and $\mathrm{Cu}^{2+}$ respectively. In order to obtain single crystals of high quality, purification is an important step. Hence, recrystallization was carried at least two or three times.

\section{Solubility}

Solubility of BTZS crystals has been determined for five temperatures $30^{\circ} \mathrm{C}, 35^{\circ} \mathrm{C}, 40^{\circ} \mathrm{C}, 45^{\circ} \mathrm{C}$ and $50^{\circ} \mathrm{C}$. Recrystalized salt is used for this purpose. A $250 \mathrm{ml}$ glass beaker containing $100 \mathrm{ml}$ of deionized water was placed in the temperature bath. The initial temperature of the bath was set at $30^{\circ} \mathrm{C}$. The beaker was closed with a sheet and placed in the magnetic stirrer. The synthesized salt was added in small amount and the stirring of the solution was continued till the formation of precipitate, which confirmed the saturation of the solution. The stirring further confirmed to have uniform temperature and concentration throughout the entire volume of the solution. On reaching the saturation, the equilibrium concentration of the solution was 
analyzed gravimetrically. The same procedure was repeated for the doped BTZS crystals. Figure 1 shows the solubility curves of pure, $\mathrm{Cd}^{2+}$ and $\mathrm{Cu}^{2+}$ doped BTZS single crystals.

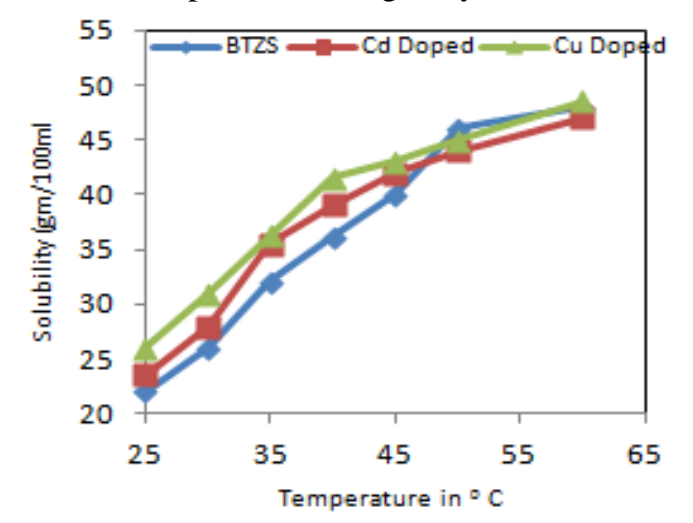

Fig. 1 Solubility curves for pure and doped BTZS

\section{Growth of pure, $\mathrm{cd}^{2+}$ and $\mathrm{cu}^{2+}$ doped btzs single crystals}

Single crystals of pure, $\mathrm{Cd}^{2+}$ and $\mathrm{Cu}^{2+}$ doped BTZS were grown by slow evaporation technique at room temperature. Saturated solutions of pure and doped BTZSwere prepared using recrystallized salt at room temperature. The solutions were filtered using filter paper and kept at room temperature to evaporate the solvent. Transparent and good quality seeds were selected for the growth experiments. The period of growth ranged between 3-4 weeks. The photographs of pure and doped BTZS single crystals are shown in Fig. 2 It is seen from the photographs that both pure and doped crystals are optically highly transparent

\subsection{X-ray diffraction studies}

\section{Characterization}

To confirm crystallinity of the grown crystals and also to determine the unit cell parameters the Xray diffraction analysis was carried out using an X-ray diffractometer model Philips Powder X-ray diffractometer using $\operatorname{CuK} \alpha\left(\lambda=1.5418 \mathrm{~A}^{\circ}\right)$. The samples were scanned over the range $20^{\circ}$ to $60^{\circ}$ at a scan rate of $1 \% \mathrm{~min}$. The unit cell dimensions were calculated using PROSZKI software package [12]. The study revealed that BTZS crystal belongs to orthorhombic crystal system. The obtained crystallographic data are presented in the Table. 1

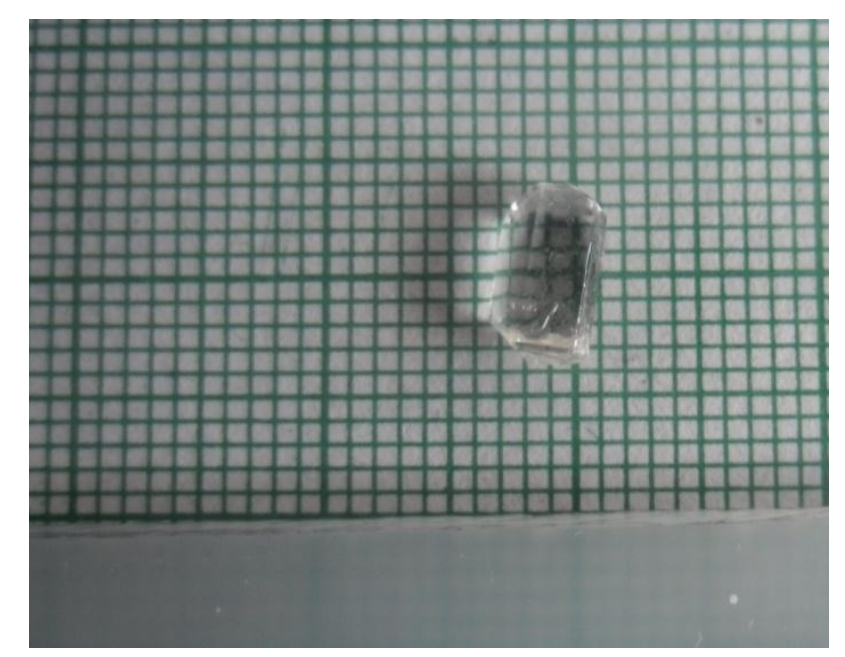

(a) 


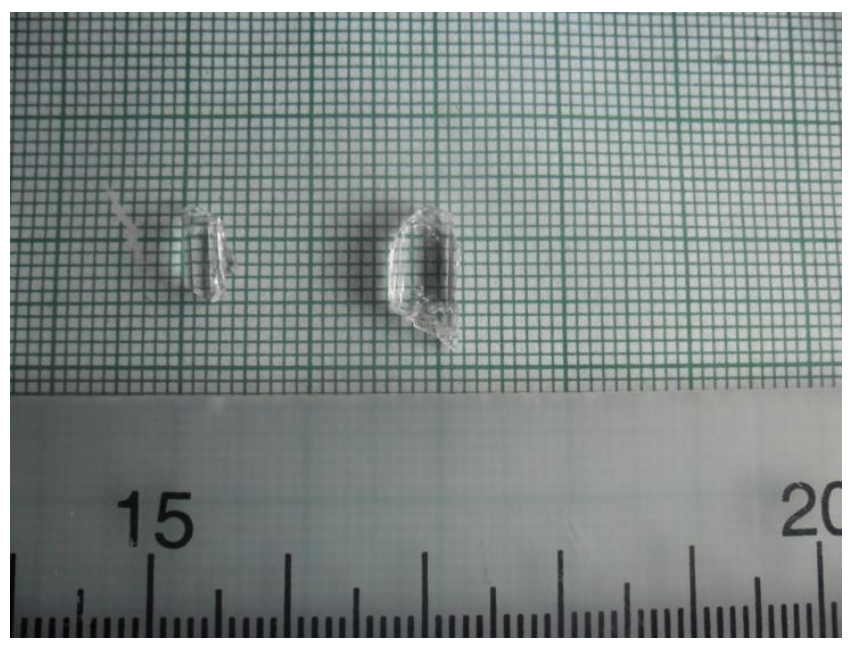

(b)

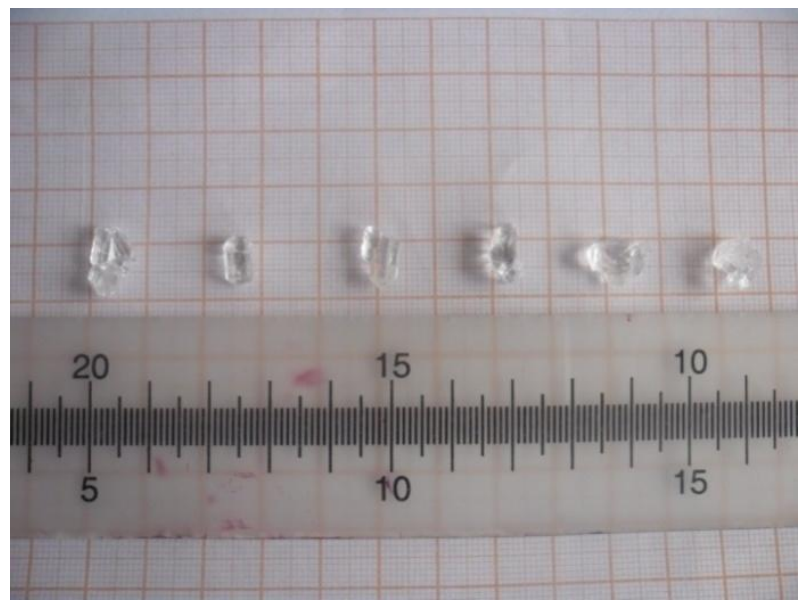

(c)

Fig. 2 Photographs of as grown (a) pure, (b) $\mathrm{Cd}^{2+}$ and (c) $\mathrm{Cu}^{2+}$ doped BTZS single crystals

\subsection{Atomic absorption spectroscopy (AAS)}

The incorporation of dopants $\left(\mathrm{Cd}^{2+}\right.$ and $\left.\mathrm{Cu}^{2+}\right)$ in doped BTZS crystals has been quantified by the atomic absorption spectroscopy (AAS) using a Varian AA100 Atomic Absorption Spectrometer. In order to determine the exact mole percentage of dopants incorporated in the doped crystals, crystalline powders of $110 \mathrm{mg}$ of $\mathrm{Cd}^{2+}$ doped BTZS and $250 \mathrm{mg}$ of $\mathrm{Cu}^{2+}$ doped BTZS were dissolved separately in $100 \mathrm{ml}$ of double distilled water. These solutions were subjected to AAS. The results revealed that dopants are very low in percentile for 1 mole $\%$ metal concentrations added and they are suitably present in the crystal lattice of grown BTZS crystals.

Table. 1 Crystal data of pure and doped BTZS single crystals

\begin{tabular}{|c|c|c|c|}
\hline $\begin{array}{c}\text { Crystallographi } \\
\text { c data }\end{array}$ & Pure-BTZS & $\mathrm{Cd}^{2+}-\mathrm{BTZS}$ & $\mathrm{Cu}^{2+}-\mathrm{BTZS}$ \\
\hline $\mathrm{a}(\AA)$ & 5.4937 & 5.484 & 5.3752 \\
\hline $\mathrm{b}(\AA)$ & 7.6383 & 8.533 & 8.4432 \\
\hline$c(\AA)$ & 8.5268 & 7.813 & 7.6415 \\
\hline Volume $(\AA)^{3}$ & 357.806 & 365.59 & 346.803 \\
\hline$\alpha$ & $90^{\circ}$ & $90^{\circ}$ & $90^{\circ}$ \\
\hline $\bar{\beta}$ & $90^{\circ}$ & $90^{\circ}$ & $90^{\circ}$ \\
\hline$\gamma$ & $90^{\circ}$ & $90^{\circ}$ & $90^{\circ}$ \\
\hline Crystal system & Orthorhombic & Orthorhombic & Orthorhombic \\
\hline
\end{tabular}

www.iosrjournals.org 


\subsection{Optical absorption studies}

The optical absorption spectra of pure, $\mathrm{Cd}^{2+}$ and $\mathrm{Cu}^{2+}$ doped BTZS crystals were recorded in the range 200-2500 nm using a Varian Cary 5E model spectrophotometer. Figures 3-5 show the optical absorption spectra of pure, $\mathrm{Cd}^{2+}$ and $\mathrm{Cu}^{2+}$ doped BTZScrystals respectively. The spectra show that pure and doped BTZS have good transmission in the entire visible region. The lower cut-off wavelength lies below $250 \mathrm{~nm}$.Efficient NLO crystals have an optical transparency lower cutoff wavelength between 200 and 400 $\mathrm{nm}$ [13].From the UV-Vis spectral analysis it is clear that there is no significant absorption in the UV and visible region thereby confirming the advantages of the crystals, the large transmission in the entire visible region and short cut off wavelength enable the crystals to be useful for second and third harmonic generation of Nd:YAG laser fundamental.The percentage of absorption of doped BTZS crystals is less than that of pure one, which is likely to improve the optical property of BTZS.

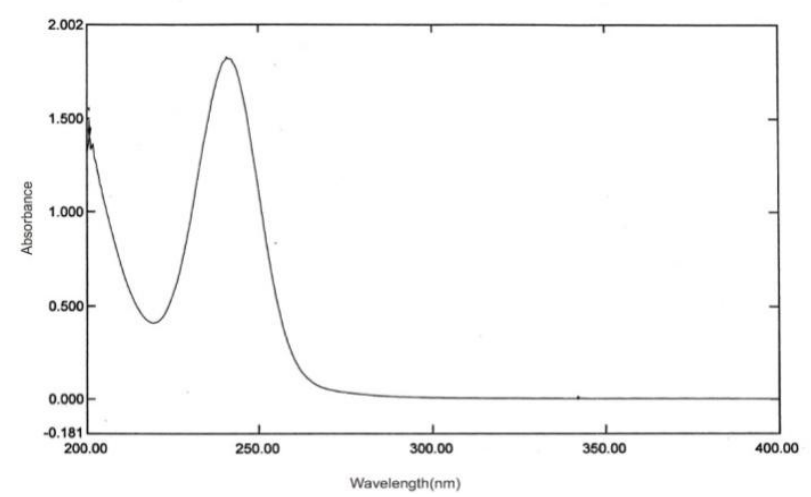

Fig. 3 The optical absorption spectrum of pure BTZScrystal

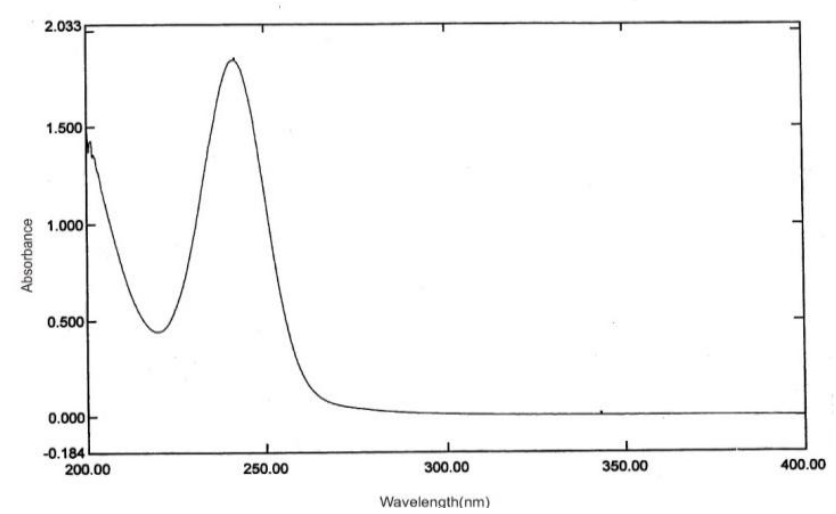

Fig. 4 The optical absorption spectrum of $\mathrm{Cd}^{2+}$ doped BTZScrystal

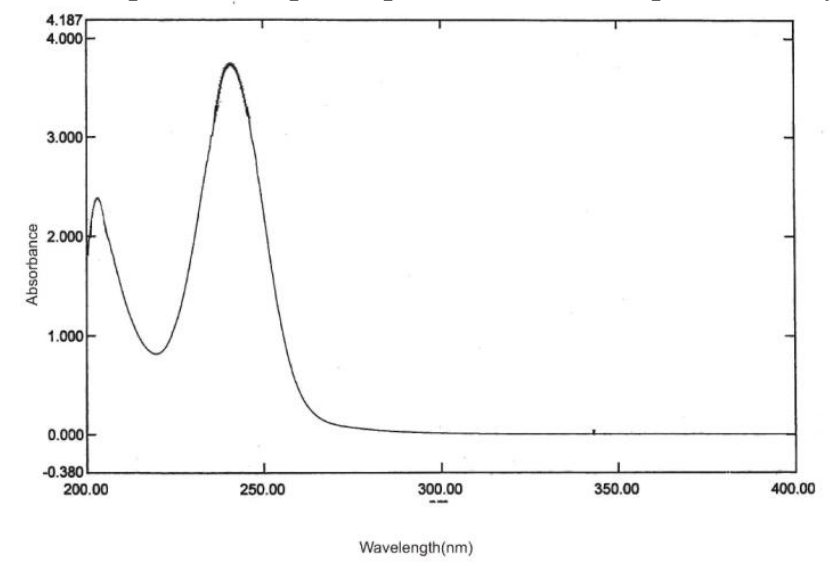

Fig. 5 The optical absorption spectrum of $\mathrm{Cu}^{2+}$ doped BTZScrystal 


\subsection{FTIR studies}

Pure, $\mathrm{Cd}^{2+}$ and $\mathrm{Cu}^{2+}$ doped BTZS crystals were subjected to the FTIR studies. The various functional groups present in BTZS crystals were identified and confirmed by the FTIR study. The spectrum was recorded in the range $4000-400 \mathrm{~cm}^{-1}$ using BRUKER IFS - $66 \mathrm{~V}$ spectrometer, by $\mathrm{KBr}$ pellet technique. Figures 6-8 show the FTIR spectra of pure, $\mathrm{Cd}^{2+}$ and $\mathrm{Cu}^{2+}$ doped BTZS crystals respectively.

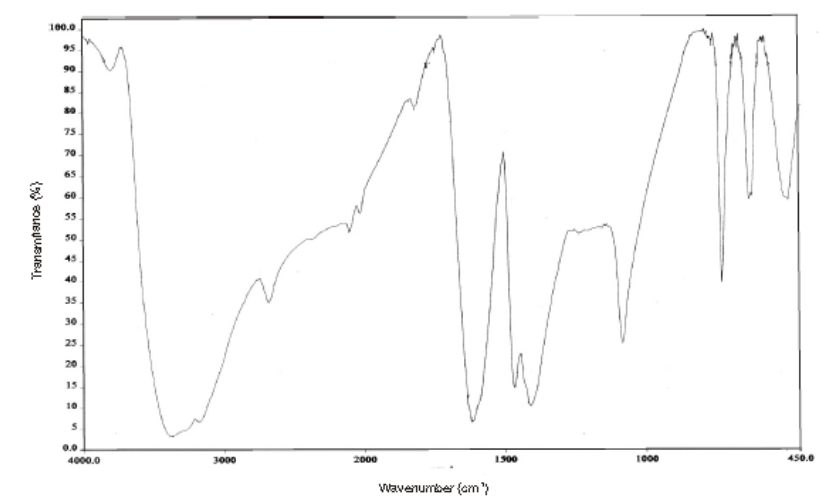

Fig. 6 The FTIR spectrum of pure BTZS crystal

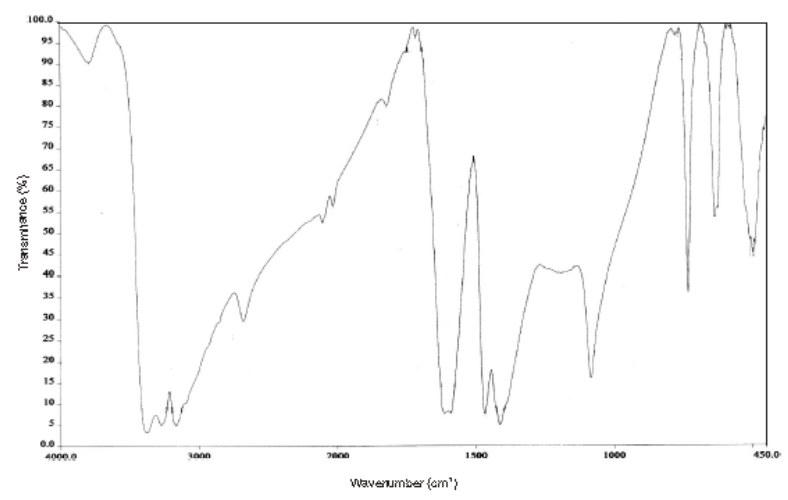

Fig. 7 The FTIR spectrum of $\mathrm{Cd}^{2+}$ doped BTZS crystal

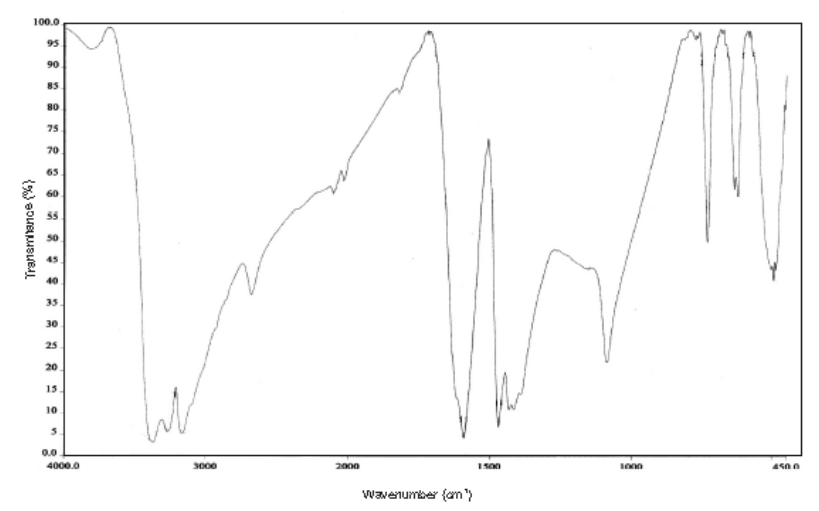

Fig. 8 The FTIR spectrum of $\mathrm{Cu}^{2+}$ doped BTZS crystal

Thiourea could form metal complexes by coordinate bonds through sulphur or nitrogen. Thiourea exhibits characteristic peaks at $1627,1472,1417,1089,740$ and $494 \mathrm{~cm}^{-1}[14]$. The band at $1627 \mathrm{~cm}^{-1}$ is due to $\mathrm{NH}_{2}$ bending. The $\mathrm{CN}$ asymmetric and symmetric stretching vibrations are observed at 1089 and $1472 \mathrm{~cm}^{-}$ ${ }^{1}$ respectively. The CS asymmetric stretching vibration is observed at $1417 \mathrm{~cm}^{-1}$. The CS symmetric stretching is observed at $740 \mathrm{~cm}^{-1}$. The band at $494 \mathrm{~cm}^{-1}$ is due to asymmetric NCN bending. If the bonding is through sulphur, there will be a decrease in the CS stretching frequency and an increase in the CN stretching frequency. The reverse happens if it is through nitrogen. The standard IR band frequencies of thiourea, and that obtained for pure and doped BTZS crystals are compared along with their assignments. 
The CN stretching (1089 and $1472 \mathrm{~cm}^{-1}$ ) frequencies of thiourea are shifted to higher frequencies of pure and doped BTZS (above 1046 and $1414 \mathrm{~cm}^{-1}$ ) and CS stretching frequencies $\left(1417\right.$ and $740 \mathrm{~cm}^{-1}$ ) are shifted to lower frequencies of pure and doped BTZS (below 1356 and below $700 \mathrm{~cm}^{-1}$ ). These observations suggest that the metals coordinate with thiourea through sulphur. The broad envelope positioned between 2750 and $3500 \mathrm{~cm}^{-1}$ corresponds to the symmetric and asymmetric stretching modes of $\mathrm{NH}_{2}$ grouping of pure and doped BTZS crystals.

\subsection{NLO Test -Kurtz Powder SHG technique}

Kurtz [15] second harmonic generation ( $\mathrm{SHG}$ ) test was performed to find the NLO property of the BTZS crystals. Kurtz technique is used as a screening technique to identify the materials with the capacity for phase matching in addition to identifying the materials with non Centro -symmetric crystal structure. The crystals were ground into powder and densely packed in between two glass slides. An Nd: YAG laser beam of pulse width $8 \mathrm{~ns}$ at a wavelength of $1064 \mathrm{~nm}$ and $10 \mathrm{~Hz}$ fundamental radiation was made to fall normally on the sample cell. The emission of green light confirms the second harmonic generation of the crystals. With an energy of the $9.2 \mathrm{~mJ}$ of input pulses, the second harmonic signal $(532 \mathrm{~nm}$ ) of $276 \mathrm{~mW}$ (pure BTZS), 287 $\mathrm{mW}\left(\mathrm{Cd}^{2+}\right.$ doped BTZS) and $294 \mathrm{~mW}\left(\mathrm{Cu}^{2+}\right.$ doped BTZS) were obtained with reference to KDP crystal (445 $\mathrm{mW})$. The experimental results indicate that the metallic dopants $\left(\mathrm{Cd}^{2+}\right.$ and $\left.\mathrm{Cu}^{2+}\right)$ slightly improve the SHG efficiency of the BTZS crystals.

Table. 2 Wavenumber assignment of thiourea, pure, $\mathrm{Cd}^{2+}$ and $\mathrm{Cu}^{2+}$ doped BTZS

\begin{tabular}{|c|c|c|c|c|}
\hline \multicolumn{4}{|c|}{ Wavenumber $\left(\mathrm{cm}^{-1}\right)$} & \multirow{2}{*}{ Assignment } \\
\hline Thiourea & Pure-BTZS & $\mathrm{Cd}^{2+}-\mathrm{BTZS}$ & $\mathrm{Cu}^{2+}-\mathrm{BTZS}$ & \\
\hline 494 & 488 & & 489 & $(\mathrm{~N}-\mathrm{C}-\mathrm{N})$ Asymmetric bending \\
\hline 740 & & 701 & 700 & $\begin{array}{c}\text { (CS) } \\
\text { Symmetric stretching }\end{array}$ \\
\hline 1089 & 1011 & 1040 & 1043 & $\begin{array}{c}(\mathrm{CN}) \\
\text { Symmetric stretching }\end{array}$ \\
\hline 1417 & & 1356 & 1356 & $\begin{array}{c}(\mathrm{CS}) \\
\text { Asymmetric stretching }\end{array}$ \\
\hline 1472 & 1460 & 1414 & 1466 & $\begin{array}{c}(\mathrm{CN}) \\
\text { Asymmetric stretching }\end{array}$ \\
\hline 1627 & 1566 & 1630 & 1510 & $\left(\mathrm{NH}_{2}\right)$ Asymmetric bending \\
\hline $3100-3300$ & 3068 & 3052 & 3051 & $\left(\mathrm{NH}_{2}\right)$ Symmetric stretching \\
\hline
\end{tabular}

\section{Conclusion}

Single crystals of pure, $\mathrm{Cd}^{2+}$ and $\mathrm{Cu}^{2+}$ doped BTZS were grown from aqueous solutions by slow solvent evaporation method. The crystal is confirmed with X-ray diffraction analysis, the crystal belongs to the orthorhombic system. The exact percentage of metallic dopants in doped BTZS was analyzed by AAS. Optical absorption studies show that the sample is optically transparent over wide wavelength region, no absorbance and good optical transmittance in the entire visible region. The non linear optical study confirms the SHG property of the crystals. The FTIR analysis confirms the bonding interaction between zinc and sulphur atoms of thiourea molecule. The promising crystal growth characteristics and properties of BTZS crystal makes it suitable for photonic and device applications.

\section{References}

[1] Zhang N., JiangM.H., YuanD.R., XuD., TaoX.T., ShaoZ.S., J.Cryst. Growth 102 (1990) 581.

[2] MonacoS.B., DavisL.E., VelskoS.P., WangandF.T., EimerlD., J.Cryst. Growth 85 (1987) 252.

[3] Uma B. Ramabadran, David E. Zelmon, Gretchen C. Kennedy,Appl. Phys. Lett. 60 (1992) 2589.

[4] MarcyH.O., WarrenL.F., WebbM.S., EbbersC.A., VelskoS.P., KennedyG.C., CatellaG.C., Appl. Opt. 31 (1992) 5051.

[5] KotlerZ., HierleR., JosseD., ZyssJ., MasseR., J. Opt. Soc. Am. B9(1992) 534.

[6] HoriuchiN., LefaucheuxF., IbanezA., JosseD., ZyssJ., Opt. Mater.12 (1999) 351. 
[7] VenkataramananV., SubramanianC.K., BhatH.L., J. Appl. Phys.77 (1995) 6049.

[8] WarrenL.F., AllredR.E., MartinezR.J., Wischmann K.B. (Eds.), Proceedings of the Fourth International Sample Electronics Conference, Society for the Advancement of Material and Process Engineering,Covina, CA, 4 1990, p. 388.

[9] ChangT.Y., CopperD.E., BurdgeG.L., Polakdigds P., Lowe-MaC.K., C.Y. ChiangC.K., ChaikanP.M., CowanD.O. (Eds.), Advanced Organic Solid Sate Materials, Materials Research Society Symposium Proceedings, Materials Research Society, Pittsburg, PA, 173, 1990, p.557

[10] DhumaneN.R., HussainiS.S., NawarkheleV.V. and ShirsatM.D. Cryst. Res. Technol.41, (2006) 897901

[11] RamajothiJ., DhanushkodiS. and NagarajanK. Cryst. Res. Technol.39, (2004) 414-420

[12] Lasocha W. and LewinskiK., J.Appl. Crystallography, 27, 437, (1994)

[13] Le Fur Y., Masse R., Cherkaoui M.Z., Nicoud J.F., Z.Kristallogr. 210 (1995) 856

[14] SilversteinR.M., Clayton Basseler G. and Morrill T.C., Spectrometric Identification of Organic Compounds, V-Ed. John Wiley and Sons, Inc. New York 1998.

[15] KurtzS.K., $\quad$ PerryT.T., $\quad$ J. $\quad$ Appl. $\quad$ Phys. 3798. 